\title{
Évaluation de la surveillance de l'infection tuberculeuse latente dans la région de Peel, en Ontario, 2010 à 2014
}

\author{
JA Majerovich ${ }^{1,2}$, L Fernandes², M Varia ${ }^{2 \star}$
}

\section{Résumé}

Contexte : Au Canada, I'identification et la prise en charge des personnes présentant une infection tuberculeuse latente (ITL) sont essentielles pour la prévention de l'évolution de l'infection tuberculeuse latente en tuberculose active. Dans la région de Peel, une grande municipalité de l'Ontario où la moitié de la population est née à l'étranger, les données de surveillance de l'infection tuberculeuse latente sont également cruciales pour la compréhension de l'épidémiologie locale de tuberculose.

Objectif : Évaluer l'utilité et la qualité des données de surveillance de l'infection tuberculeuse latente recueillies par l'intermédiaire du Système d'information sur la santé publique intégré (SISP-i) de 2010 à 2014, et formuler des recommandations dans le but d'améliorer la surveillance dans la région de Peel.

Méthodologie : Utilisation du cadre du Centre européen de prévention et de contrôle des maladies (ECDC) pour l'évaluation de la surveillance. L'évaluation de la qualité des données portait sur l'exhaustivité et la validité des variables clés de la base de données du SISP-i. L'utilité des données de surveillance dans l'orientation des processus décisionnels des programmes a été évaluée dans le cadre d'entrevues avec les intervenants du bureau de santé publique de Peel.

Résultats : Sur 6576 registres du SISP-i évalués, les données relatives au sexe et à la date de naissance étaient complétées à plus de $99 \%$, tandis que plus de la moitié des champs dédiés aux facteurs de risque étaient vides ou portaient la mention " inconnu ». Une comparaison de 192 dossiers papier aux registres correspondants du SISP-i a permis d'identifier des erreurs de codage dans plus de $40 \%$ des champs dédiés aux facteurs de risque du SISP-i. L'achèvement du traitement décrit dans le SISP-i (20\%) était inférieur aux données obtenues lors d'une enquête téléphonique de suivi des cas (50\%). Les intervenants ont jugé les données de surveillance utiles (100\%). Toutefois, ils ont formulé des recommandations visant à améliorer I'analyse et la collecte des données.

Conclusion : L'évaluation de la surveillance de l'infection tuberculeuse latente à des fins d'amélioration de l'utilité et de la qualité des données pour la planification de programmes est essentielle pour l'éradication de la tuberculose. Cette évaluation a donné lieu à la standardisation des processus de saisie de données et à la poursuite du suivi direct auprès des patients atteints d'une infection tuberculeuse latente pour confirmer l'achèvement du traitement. Des efforts sont actuellement déployés pour tenter de comprendre les obstacles à l'amorce et à l'achèvement du traitement.

\author{
Affiliations \\ ${ }^{1}$ Division de la formation et de \\ l'intervention pour le service sur \\ le terrain de l'Agence de la santé \\ publique du Canada, Ottawa \\ (Ontario) \\ ${ }^{2}$ Bureau de santé publique de \\ Peel, Mississauga, (Ontario)
}

*Correspondance : monali.varia@ peelregion.ca

Citation proposée : Majerovich JA, Fernandes L, Varia M. Évaluation de la surveillance de l'infection tuberculeuse latente dans la région de Peel, en Ontario, 2010 à 2014. Relevé des maladies transmissibles au Canada. 2017;43(5):127-32. https://doi.org/10.14745/ccdr.v43i05a06f

\section{Introduction}

L'infection à Mycobacterium tuberculosis peut donner lieu à une infection tuberculeuse latente (ITL) ou à une tuberculose active (1). L'évolution de l'infection tuberculeuse latente en tuberculose active peut être réduite de $90 \%$ maximum grâce à un traitement préventif de neuf mois $(1,2)$. L'Organisation mondiale de la Santé a déterminé qu'une meilleure identification et une meilleure prise en charge des personnes atteintes d'une infection tuberculeuse latente présentant un risque plus élevé d'évoluer en tuberculose active sont essentielles pour permettre d'atteindre les nouveaux objectifs d'éradication de la tuberculose (3). Bien que le Canada présente une faible incidence globale de la tuberculose, les taux sont supérieurs dans les sous-populations telles que les immigrants provenant de pays où l'incidence de la maladie est élevée, les personnes voyageant à destination de ces pays et les Canadiens autochtones. La région de Peel est une grande municipalité de l'Ontario comptant 1,4 million d'habitants. La 
moitié $(50,5 \%)$ de la population de Peel est née à l'étranger, dont une grande partie dans les pays où la tuberculose est endémique. Cette proportion est supérieure au pourcentage d'habitants de l'Ontario nés à l'étranger (28,5 \%) (4). En 2014, le taux d'incidence standardisé en fonction de l'âge de la tuberculose active dans la région de Peel était de 9,1 pour 100 000, contre 4,0 pour 100000 en Ontario (5). Chaque année, près de 1200 cas d'infection tuberculeuse latente sont signalés au service de santé publique local, le bureau de santé publique de Peel (6).

L'identification et la prise en charge des personnes atteintes d'une infection tuberculeuse latente présentant un risque accru d'évoluer en tuberculose active constituent un élément clé de la stratégie de prévention de l'évolution de l'infection tuberculeuse latente en tuberculose active. Les Normes canadiennes pour la lutte antituberculeuse visent qu'au moins $80 \%$ de ces personnes présentant une infection tuberculeuse latente qui commencent le traitement prendront le nombre requis de doses (1). Les données de surveillance de l'infection tuberculeuse latente de Peel permettent de suivre l'achèvement du traitement, d'identifier les groupes de population présentant un risque accru de développer une infection active et d'évaluer l'efficacité des interventions relatives au programme.

Malgré l'importance de la surveillance dans l'évaluation de l'efficacité des efforts de prévention et de lutte contre la tuberculose, les données probantes publiées permettant d'évaluer la surveillance de l'infection tuberculeuse latente sont limitées (7-9). Les objectifs de cette étude étaient a) d'évaluer les données de surveillance de l'infection tuberculeuse latente recueillies par l'intermédiaire du Système d'information sur la santé publique intégré (SISP-i) de l'Ontario sur la base de l'évaluation de deux critères (qualité [exhaustivité et validité] et utilité des données) et b) de formuler des recommandations pour améliorer la surveillance de l'infection tuberculeuse latente dans la région de Peel.

En Ontario, le Système d'information sur la santé publique intégré (SISP-i) est la base de données utilisée par tous les services de santé publique pour signaler les cas de maladie à déclaration obligatoire (dont l'infection tuberculeuse latente) au ministère de la Santé et des Soins de longue durée, conformément à la Loi sur la protection et la promotion de la santé de l'Ontario, L.R.O. $1990(10,11)$. Les données sur les cas d'infection tuberculeuse latente sont signalées de façon passive au bureau de santé publique de Peel par des sources de déclaration locales telles que les hôpitaux et les cliniciens de la collectivité. Lorsqu'un cas d'infection tuberculeuse latente est signalé au bureau de santé publique de Peel, des infirmières en santé publique indiquent les renseignements le concernant sur un dossier papier. Ces données sont ensuite saisies dans le SISP-i. Les épidémiologistes du bureau de santé publique de Peel analysent régulièrement les données de surveillance afin d'identifier les répercussions des programmes.

\section{Méthodologie}

L'évaluation du système de surveillance de l'infection tuberculeuse latente était fondée sur le cadre du Centre européen de prévention et de contrôle des maladies (ECDC) (12). Ce cadre a été choisi pour l'accent qu'il mettait sur la qualité des données, qui était un objectif principal de l'évaluation de la surveillance. Les critères de surveillance évalués sont résumés dans le tableau 1. L'exhaustivité interne, ainsi que la validité externe et interne des données ont été choisies car les processus décisionnels des programmes sont fondés sur les renseignements disponibles dans le SISP-i. L'utilité des données a été choisie dans le but d'identifier les points forts de la surveillance et les possibilités d'améliorer l'utilisation des données dans le cadre des mesures de santé publique.

\section{Tableau 1 : Critères de surveillance évalués concernant} l'infection tuberculeuse latente

\begin{tabular}{|l|l|}
\hline \hline \multicolumn{1}{|c|}{ Critère } & \multicolumn{1}{c|}{ Définition } \\
\hline Exhaustivité interne & $\begin{array}{l}\text { Proportion de champs de données complétés } \\
\text { dans la base de données }\end{array}$ \\
\hline Validité interne & $\begin{array}{l}\text { Ampleur des erreurs dans le système de } \\
\text { surveillance, par exemple, erreurs de codage } \\
\text { dans le transfert d'un niveau du système au } \\
\text { suivant }\end{array}$ \\
\hline Validité externe & $\begin{array}{l}\text { Exactitude des renseignements consignés sur les } \\
\text { cas par rapport à une base de données externe } \\
\text { ou à une « référence » }\end{array}$ \\
\hline Utilité & $\begin{array}{l}\text { Utilité des résultats de la surveillance pour les } \\
\text { mesures de santé publique }\end{array}$ \\
\hline
\end{tabular}

Exhaustivité interne : L'exhaustivité interne a été mesurée par le calcul de la proportion de réponses manquantes (champs vides ou portant la mention « inconnu ») pour certaines variables. Il s'agit notamment des variables suivantes : caractéristiques démographiques du client (date de naissance, sexe, origine), facteurs de risque (facteurs sociaux comportementaux, exposition, facteurs de risque médicaux) et variables liées au traitement (date de début et de fin du traitement indiquée par le client ou son médecin). Les champs de données concernés de tous les cas d'infection tuberculeuse latente dans la région de Peel signalés entre 2010 et 2014 ont été extraits de la base de données du SISP-i à l'aide du logiciel Cognos ReportNet et analysés dans Stata 14.

Validité interne : Avec, pour référence, les dossiers papier du bureau de santé publique de Peel concernant les cas d'infection tuberculeuse latente, la validité interne a été évaluée par la comparaison des données du SISP-i à celles du dossier papier pour les variables en question. En raison du grand nombre de cas d'infection tuberculeuse latente, une taille d'échantillon de 203 cas d'ITL de l'année 2014 a été calculée sur la base d'un intervalle de confiance à $95 \%$, ainsi qu'une taille de population de 1 157, une fréquence hypothétique de $20 \%$ du facteur de résultat dans la population et un effet du plan de sondage de 1,0 pour un échantillon aléatoire (www.openepi.com/SampleSize/ SSPropor.htm). Un dossier sur cinq a été échantillonné à partir de 2014 et 192 dossiers ont été évalués car certains n'ont pu être immédiatement localisés.

Validité externe : La validité externe a été évaluée par comparaison des données d'achèvement du traitement contre l'infection tuberculeuse latente du SISP-i pour la période 2010 à 2014 aux données d'achèvement du traitement obtenues au cours du suivi téléphonique actif des cas d'infection tuberculeuse latente. L'enquête téléphonique sur les cas d'infection tuberculeuse latente a été réalisée par le personnel du bureau de santé publique de Peel, de juillet 2015 à avril 2016. Les personnes en question avaient débuté leur traitement. Étant donné que le traitement de première intention (1) contre l'infection tuberculeuse latente dure généralement neuf mois, l'infection tuberculeuse latente a probablement 
été diagnostiquée, chez les 208 clients contactés, en 2014 et en 2015 .

Utilité : Huit entrevues semi-structurées ont été menées en personne auprès d'intervenants internes du bureau de santé publique de Peel. L'objectif était d'évaluer l'utilité de la surveillance de l'infection tuberculeuse latente dans l'orientation des mesures de santé publique. Les intervenants ont été choisis de façon à ce que différents points de vue soient représentés : celui des infirmières en santé publique de première ligne, directement impliquées dans le fonctionnement du système, mais également celui des décideurs de la santé publique, notamment du médecin hygiéniste. Les intervenants ont été invités à donner leur opinion sur les mesures prises en fonction des données de surveillance, ainsi que sur les défis et les occasions propres à la surveillance. Leurs réponses ont été consignées en format texte. Trois épidémiologistes du bureau de santé publique de Peel (J.A.M., M.V. et un épidémiologiste spécialiste de la tuberculose) ont procédé à une analyse des données. Ils ont commencé par évaluer les thèmes, un par un, pour parvenir à un consensus concernant les thèmes communs à l'aide d'une méthode facilitée par petits groupes.

\section{Résultats}

Exhaustivité interne : Pour les 6576 cas d'infection tuberculeuse latente, les données concernant le sexe et la date de naissance des personnes étaient pratiquement complétées à $100 \%$ (tableau 2). Parmi les cas d'infection tuberculeuse latente dont l'origine était manquante ou " inconnue » $(n=1716), 0,1 \%$ mentionnaient la province de naissance et $1,9 \%$, le pays de naissance. L'exhaustivité était sous-optimale pour les champs de données suivants : date de fin de traitement (champ incomplet à $64,6 \%$ ), date de début de traitement (champ incomplet à $52,3 \%$ ) et facteur de risque (champ incomplet à 54,7 \%).

Tableau 2 : Exhaustivité interne des champs de données relatifs à l'infection tuberculeuse latente

\begin{tabular}{|c|c|c|c|c|}
\hline Variable & $\begin{array}{c}\text { Nombre } \\
\text { total } \\
\text { de cas } \\
\text { enregistrés }\end{array}$ & $\begin{array}{l}\text { Nombre } \\
\text { (\%) de cas } \\
\text { dont les } \\
\text { champs de } \\
\text { données } \\
\text { étaient } \\
\text { manquants }\end{array}$ & $\begin{array}{l}\text { Nombre (\%) } \\
\text { de cas dont } \\
\text { les champs } \\
\text { de données } \\
\text { présentaient } \\
\text { la mention } \\
\text { " inconnu " }\end{array}$ & $\begin{array}{c}\text { Nombre (\%) } \\
\text { de cas dont } \\
\text { les champs } \\
\text { de données } \\
\text { étaient } \\
\text { complétés } \\
\text { intégralement }\end{array}$ \\
\hline $\begin{array}{l}\text { Date de } \\
\text { naissance }\end{array}$ & 6576 & $0(0)$ & $0(0)$ & $6576(100)$ \\
\hline Sexe & 6576 & $0(0)$ & $46(0,7)$ & $6530(99,3)$ \\
\hline Origine & 6576 & $7(0,1)$ & $1709(26,0)$ & $4860(73,9)$ \\
\hline $\begin{array}{l}\text { Facteur de } \\
\text { risque }\end{array}$ & 6576 & $270(4,1)$ & $3326(50,6)$ & $2980(45,3)$ \\
\hline $\begin{array}{l}\text { Date de } \\
\text { début de } \\
\text { traitement }\end{array}$ & 6576 & $3436(52,3)$ & $0(0)$ & $3140(47,7)$ \\
\hline $\begin{array}{l}\text { Date de } \\
\text { fin de } \\
\text { traitement }{ }^{1}\end{array}$ & 3140 & $2030(64,6)$ & $0(0)$ & $1110(35,4)$ \\
\hline
\end{tabular}

RMTC • Le 4 mai $2017 \bullet$ Volume 43-5
Validité interne : À l'exception des données de facteur de risque, toutes les variables évaluées présentaient une validité interne élevée (> $94 \%$ ) (tableau 3). Concernant le facteur de risque, $57 \%$ des réponses relatives au SISP-i ne correspondaient pas à celles du dossier papier. Parmi ces données discordantes (concernant le facteur de risque médical), 46,3\% présentaient un facteur de risque médical " inconnu » dans le SISP-i, tandis que sur le dossier papier, le médecin du client avait spécialement indiqué l'absence de facteur de risque médical. En outre, 10,9\% des dossiers papier examinés présentaient un facteur de risque médical non consigné dans le SISP-i.

Tableau 3 : Validité interne des champs de données relatifs à l'infection tuberculeuse latente $\left(N=192^{1}\right)$

\begin{tabular}{|l|c|}
\hline Variable & $\begin{array}{l}\text { Nombre }(\%) \text { de cas présentant } \\
\text { des données valides }\end{array}$ \\
\hline Sexe & $191(99,5)$ \\
\hline Date de naissance & $190(99,0)$ \\
\hline Résultat du traitement & $190(99,0)$ \\
\hline Motif de fin du traitement & $189(98,4)$ \\
\hline Pays de naissance (immigration) & $188(97,9)$ \\
\hline Date de fin de traitement & $185(96,4)$ \\
\hline Date de début de traitement & $181(94,3)$ \\
\hline Facteur de risque & $110(57,3)$ \\
\hline Abréviation: \%, pourcentage &
\end{tabular}

${ }^{1}$ Nombre total de dossier papier examinés

Validité externe : Le suivi téléphonique actif des 208 cas d'infection tuberculeuse latente ne présentant pas la date de fin de traitement dans le SISP-i a permis de déterminer un taux d'achèvement du traitement de $50 \%$. Ceci a été comparé au taux d'achèvement du traitement consigné dans le SISP-i, qui était d'environ 20 \% entre 2010 et 2013 (tableau 4). En 2014, le taux d'achèvement du traitement contre l'infection tuberculeuse latente dans le SISP-i était de $28 \%$. Toutefois, cette valeur inclut les clients qui ont participé à l'enquête téléphonique, et dont la date de fin de traitement a ensuite été saisie dans le SISP-i. Aucune nouvelle intervention susceptible de justifier cette augmentation n'a été entreprise.

Tableau 4 : Validité externe des taux d'achèvement du traitement contre l'infection tuberculeuse latente

\begin{tabular}{|c|r|r|}
\hline \hline Année & $\begin{array}{c}\text { Nombre total de cas } \\
\text { ayant commencé le } \\
\text { traitement }\end{array}$ & $\begin{array}{c}\text { Nombre (\%) de cas } \\
\text { dont l'achèvement du } \\
\text { traitement est indiqué } \\
\text { dans le SISP-i }\end{array}$ \\
\hline 2010 & 723 & $141(19,5)$ \\
\hline 2011 & 698 & $149(21,3)$ \\
\hline 2012 & 589 & $118(20,0)$ \\
\hline 2013 & 602 & $115(19,1)$ \\
\hline 2014 & 528 & $149(28,2)$ \\
\hline
\end{tabular}

Utilité : Le taux de réponse des entrevues avec les intervenants clés était de $100 \%$. Globalement, les données de surveillance ont été jugées utiles à la planification et à la mise en œuvre des programmes (100\%). Toutefois, l'évaluation a permis de dégager trois thèmes s'accompagnant de recommandations destinées 
à améliorer l'utilité des données de surveillance de l'infection tuberculeuse latente :

1. La surveillance passive, qui dépend de l'envoi, par les cliniciens, des formulaires complétés, constitue un obstacle à l'exhaustivité des renseignements sur les facteurs de risque et l'achèvement du traitement. Une personne interrogée a indiqué que « la qualité (l'exhaustivité) des données est conforme à la qualité des données que nous recevons des médecins... nous constatons des lacunes au niveau des données relatives aux facteurs de risque et à l'achèvement du traitement ». Parmi les stratégies proposées pour améliorer la qualité des données transmises par les cliniciens de la collectivité, citons le système d'alerte relative au dossier médical électronique. Une autre personne interrogée a expliqué les conséquences de ces données incomplètes sur la capacité à élaborer des interventions efficaces de prévention et de lutte contre l'infection. " (Il existe des) lacunes dans les déclarations des médecins et au niveau de l'exhaustivité des formulaires de surveillance... nous ne savons pas quelle population cibler et surveiller de plus près. "

2. Le personnel du bureau de santé publique de Peel utilise les données de surveillance pour orienter les mesures de santé publique. Une personne interrogée a déclaré : " un rapport de surveillance a mis en évidence des taux élevés de tuberculose active et un faible taux de cas signalés d'infection tuberculeuse latente dans la municipalité de Peel... les infirmières spécialistes de la tuberculose ont donc mené des activités de sensibilisation auprès des médecins afin qu'ils sachent quelle population cibler dans le cadre du dépistage afin d'améliorer le diagnostic de l'infection tuberculeuse latente » (pour détecter et traiter les cas d'infection tuberculeuse latente, et éviter ainsi que l'infection évolue en tuberculose active).

3. Il est possible de renforcer l'utilité des données de surveillance de l'infection tuberculeuse latente pour la prise de mesures en améliorant l'analyse des données et la collecte de données en fonction du contexte local. Une personne interrogée a posé les questions suivantes : «Dans quelle mesure les données sont-elles conformes à la réalité de l'infection tuberculeuse latente dans la région de Peel? Les cas de tuberculose active dans la région de Peel proviennent-ils de cas connus d'infection tuberculeuse latente (et dans lesquels il était possible d'intervenir)?" Une autre personne interrogée a indiqué que les politiques actuelles en matière de dépistage des immigrants exigent de nombreuses ressources pour « un risque de transmission qui n'est pas considérable (dans la région de Peel); il convient d'adapter les interventions relatives à la tuberculose au contexte local ».

\section{Discussion}

L'évaluation de la surveillance de l'infection tuberculeuse latente dans la région de Peel a permis d'identifier une grande exhaustivité (>99\%) des variables démographiques, une excellente validité (> $99 \%$ ) de la plupart des champs du SISP-i et un achèvement du traitement contre l'infection tuberculeuse latente supérieur à ce qui a été indiqué dans le SISP-i. Toutefois, moins de la moitié des données concernant les facteurs de risque du SISP-i sont complètes et un peu plus de la moitié de ces données sont valides. Ce constat met en évidence les limites du SISP-i et la nécessité de modifier les processus de saisie des données et de gestion des cas. Néanmoins, la surveillance de l'infection tuberculeuse latente dans la région de Peel s'est avérée utile dans l'orientation des mesures de santé publique, telles que les interventions de sensibilisation auprès des médecins.

Plusieurs possibilités d'amélioration de la qualité des données ont été identifiées. Avec $54,7 \%$ de données sur les facteurs de risque de l'infection tuberculeuse latente manquantes ou " inconnues", il convient de standardiser les processus de saisie de données. En l'absence de champ où préciser « aucun facteur de risque médical ", ce renseignement a été indiqué comme " inconnu » dans le SISP-i, ce qui explique en partie les discordances. Toutefois, $10,9 \%$ des cas d'infection tuberculeuse latente présentaient un facteur de risque médical dans le dossier papier, non consigné dans le SISP-i. L'exactitude des données sur les facteurs de risque saisies dans le SISP-i est particulièrement importante du point de vue coûts-avantages car des ressources pourraient être mobilisées pour suivre les cas d'infection tuberculeuse latente présentant un risque accru d'évoluer en tuberculose active (par exemple, en raison d'un facteur de risque médical). La validité et l'exhaustivité des données sur les facteurs de risque sont absolument nécessaires. Elles permettront d'identifier les groupes de population présentant un risque accru et nécessitant des mesures ciblées.

La question du pourcentage élevé $(64,6 \%)$ de données sur la date de fin de traitement manquantes ou " inconnues » est en train d'être traitée. Le système actuel passif de déclaration des médecins contribue au faible pourcentage de données renseignées sur l'achèvement du traitement contre l'infection tuberculeuse latente. Le suivi téléphonique actif des cas d'infection tuberculeuse latente par le personnel du bureau de santé publique permet de mieux saisir les données sur l'achèvement du traitement; toutefois, avec plus de 1200 cas d'infection tuberculeuse latente signalés dans la région de Peel chaque année, cette méthode n'est pas viable. À plus long terme, la mise au point de solutions technologiques à même de faciliter la déclaration, par les cliniciens, des facteurs de risque et de l'achèvement du traitement au bureau de santé publique est à l'étude. II serait également utile d'inviter les cliniciens de la collectivité à participer à une consultation avec les intervenants externes afin qu'ensemble, ils puissent déterminer comment résoudre les problèmes qu'ils rencontrent dans la déclaration des données relatives à l'infection tuberculeuse latente.

L'un des points forts de cette évaluation repose sur les recommandations qu'elle a permis de formuler en vue de l'amélioration de l'utilité des données de surveillance, comme la réalisation d'analyses spécifiques permettant de mieux comprendre la population touchée par l'infection tuberculeuse latente dans la région de Peel. Par exemple, les intervenants internes ont abordé le rôle du bureau de santé publique dans la prise en charge des cas d'infection tuberculeuse latente détectés lors du dépistage de la tuberculose auprès des nouveaux immigrants au Canada. Ce thème, qui s'est dégagé des entrevues réalisées, a incité la municipalité de Peel à réanalyser les données disponibles sur la tuberculose à partir de 2015. Elle a ainsi constaté que $23 \%$ des personnes atteintes de tuberculose nées à l'étranger ont reçu le diagnostic un à cinq ans après avoir immigré au Canada, tandis que $40 \%$ des cas de tuberculose active sont diagnostiqués après plus de 15 ans passés au Canada (données non publiées). Bien que plusieurs raisons peuvent expliquer cet état de fait (par exemple, développement d'affections chroniques augmentant le risque de réactivation, ou retour dans le pays de naissance donnant lieu à une réexposition à la tuberculose), le risque de développer la tuberculose pour les cas de la région de Peel n'est pas maximal au moment de l'immigration des personnes au Canada et de l'intervention des autorités de santé publique. D'autres analyses visant à 
examiner les différences entre les personnes atteintes d'infection tuberculeuse latente qui achèvent leur traitement et celles qui ne le terminent pas sont également en cours.

Deux limites influent sur les résultats de l'évaluation. Dans l'idéal, dans le cadre de l'évaluation de la validité externe, les données de surveillance devraient être mesurées par rapport à une référence. Les auteurs de cet article n'avaient pas accès aux dossiers des patients atteints d'infection tuberculeuse latente tenus par les cliniciens de la collectivité. Par conséquent, ils ont eu recours, à titre de référence, à une enquête téléphonique sur les cas d'infection tuberculeuse latente afin de déterminer si le traitement avait été achevé. Si le suivi téléphonique du bureau de santé publique de Peel auprès d'un échantillon de cas d'infection tuberculeuse latente a mis en évidence les lacunes dans le signalement de l'achèvement du traitement dans le SISP-i, l'ampleur de ces lacunes n'a toutefois pas pu être quantifiée. Toutefois, il existe aussi un risque que les personnes atteintes d'infection tuberculeuse latente ne soient pas conscientes qu'elles n'ont pas terminé le traitement intégral, d'une durée de neuf mois. L'auto-déclaration de l'achèvement du traitement peut donc avoir donné lieu à une surestimation. La seconde limite réside dans l'incapacité à évaluer l'exhaustivité (ou la sensibilité) externe, c'est-à-dire la mesure dans laquelle les cas d'infection tuberculeuse latente de la région de Peel ont été saisis par le système de surveillance actuel. Une étude portant sur la surveillance de l'infection tuberculeuse latente dans l'État du Massachusetts (États-Unis) a mis en évidence une probable sous-estimation de l'infection tuberculeuse latente, ainsi que l'insuffisance du caractère obligatoire du signalement dans la détection de l'infection tuberculeuse latente (7). Les auteurs suggèrent d'améliorer le dépistage ciblé, ou de procéder à une surveillance active de l'infection tuberculeuse latente ou à une surveillance en laboratoire pour éradiquer la tuberculose aux États-Unis. Ces stratégies peuvent également s'appliquer à la région de Peel.

L'exactitude des données de surveillance de l'infection tuberculeuse latente, ainsi que leur utilité dans l'orientation des mesures de santé publique sont essentielles pour la prévention et la lutte contre la tuberculose. Les Normes canadiennes pour la lutte antituberculeuse visent qu'au moins $80 \%$ des personnes présentant une infection tuberculeuse latente qui commencent un traitement doivent l'achever. Au cours de cette évaluation, le suivi téléphonique réalisé auprès des cas d'infection tuberculeuse latente de la région de Peel a permis d'identifier un taux d'achèvement du traitement de $50 \%$, un taux nettement inférieur à l'objectif national. Tandis que le bureau de santé publique poursuit sa surveillance active de façon à établir les taux réels d'achèvement du traitement contre l'infection tuberculeuse latente, des efforts sont également déployés pour tenter de comprendre les obstacles à l'amorce et à l'achèvement de ce traitement dans la région de Peel. Cette évaluation de la surveillance devrait s'appliquer à d'autres provinces et territoires du Canada, ainsi qu'à d'autres pays développés, en particulier ceux qui accueillent des sous-populations présentant des taux supérieurs de tuberculose active. L'évaluation et l'amélioration de la surveillance de l'infection tuberculeuse latente sont essentielles pour faire progresser les efforts d'éradication de la tuberculose au Canada.

\section{Déclarations des auteurs}

J.A.M. - Conceptualisation, méthodologie, analyse officielle, enquête, rédaction de l'ébauche originale, examen et révision L.F. - Conceptualisation, rédaction-examen et révision M.V. - Conceptualisation, méthodologie, rédaction-examen et révision, supervision

\section{Conflit d'intérêts}

Aucun.

\section{Remerciements}

Les auteurs souhaitent remercier les cliniciens de la collectivité de Peel, qui ont transmis les données sur l'infection tuberculeuse latente. Ils remercient également, au sein du bureau de santé publique de Peel, le personnel du programme dédié à la tuberculose, M. Baltazar, S. Raju, G. Kujbida, M. Horn, A. Richards, R. Strange, I. Mogck, K. Bingham et M. Hau. Ils remercient également, au sein de l'Agence de la santé publique du Canada, F.W. Tremblay, D. Paquette, M. Wood, N. Gilbert, M. McGuire, la Division de la formation et de l'intervention pour le service sur le terrain et le Programme canadien d'épidémiologie de terrain Cohorte 41

\section{Financement}

Ces travaux ont bénéficié du soutien du bureau de santé publique de Peel et du Programme canadien d'épidémiologie de terrain de l'Agence de la santé publique du Canada.

\section{Références}

1. Agence de la santé publique du Canada, Société Canadienne de thoracologie. Normes canadiennes pour la lutte antituberculeuse $7^{\mathrm{e}}$ édition, 2013 [Internet]. Ottawa: ASPC; 2014 [consulté le 25 fév 2017]. Disponible à l'adresse : http://www.lignesdirectricesrespiratoires.ca/sites/all/files/ NCLA_FR_7_edition.pdf.

2. Kiazyk S, Ball TB. L'infection tuberculeuse latente : un aperçu. Relevé des maladies transmissible au Canada. 2017;43(3):626. Disponible à l'adresse : http://www.phac-aspc.gc.ca/ publicat/ccdr-rmtc/17vol43/dr-rm43-3-4/ar-01-fra.php.

3. Organisation mondiale de la Santé. Mise en œuvre de la stratégie pour mettre fin à la tuberculose : point essentiels [Internet]. Genève: OMS; 2015 [consulté le 3 mars 2017]. Disponible à l'adresse : www.who.int/tb/publications/2015/ The_Essentials_to_End_TB/fr/.

4. Statistique Canada. Enquête nationale auprès des ménages (ENM) [Internet]. Ottawa: Statistique Canada [mise à jour le 05 oct 2011; consulté le 15 mars 2017]. Disponible à l'adresse : http://www23.statcan.gc.ca/imdb/p2SV_f.pl?Funct ion=getSurvey\&SDDS=5178.

5. Santé publique Ontario. Incidence du fardeau des maladies infectieuses à déclaration obligatoire (en anglais) [Internet]. Incidence de la tuberculose. Toronto: Santé publique Ontario [mise à jour le 15 mai 2016; consulté le 15 mars 2017]. Disponible à l'adresse : http://www.publichealthontario. 
$\mathrm{ca} / \mathrm{fr} /$ DataAndAnalytics/Snapshots/Pages/ReportableBurdensome-Infectious-Disease-Incidence.aspx.

6. Ministère de la Santé et des Soins de longue durée de I'Ontario. Integrated Public Health Information System (iPHIS) database. Toronto: Gouvernement de l'Ontario; 2017. Données extraites par Peel Public Health, 2016 Mar 08. D'autre renseignements aux : https://www.peelregion.ca/ health/statusdata/DataSources/HSD12_17.asp.

7. Hochberg N, Kubiak R, Tibbs A, Elder H, Sharnprapai S, Etkind S, Horsburgh Jr. C. Effectiveness of reporting on latent tuberculosis infection in Massachusetts, 2006-2008. Public Health Action. 2014 Mar 21;4(1):53-55.

8. Theocharopoulos G, Antoine D. Questioning the usefulness of the latent tuberculosis infection (LTBI) surveillance in children in France, 2010-2014. In: European Scientific Conference on Applied Infectious Disease Epidemiology abstract book. Stockholm: ECDC; 2016. p.112. Disponible à I'adresse : http://ecdc.europa.eu/en/ESCAIDE/programme/ abstract-book/Documents/ESCAIDE\%20Abstract\%20 Book\%202016.pdf.

9. Sanderson J, Virkud A, Trieu L, Jacobson L, Crossa A, Ahuja S. Leveraging electronic health record data to evaluate the completeness of provider reporting for latent tuberculosis infection, New York City, 2013. Poster presentation: Council of State and Territorial Epidemiologists Annual Conference proceedings. June 2016. Disponible à l'adresse : https://cste. confex.com/cste/2016/webprogram/start.html.

10. Gouvernement de I'Ontario. Protection et la promotion de la santé (loi sur la), L.R.O. 1990, chap.H.7 [consulté le 15 mars 2017]. Disponible à l'adresse : https://www.ontario.ca/fr/lois/ loi/90h07.

11. Ministère de la Santé et des Soins de longue durée. Normes de santé publique de l'Ontario: Protocole de prévention et de contrôle de la tuberculose, 2008 [Internet]. Toronto: Gouvernement de l'Ontario; 2008 [consulté le 25 fév 2017]. Disponible à l'adresse : http://www.health.gov.on.ca/fr/pro/ programs/publichealth/oph_standards/docs/tuberculosis_ prevention_controlf.pdf.

12. European Centre for Disease Prevention and Control. Technical document. Data quality monitoring and surveillance system evaluation [Internet]. Stockholm: ECDC; 2014 [consulté le 24 fév 2017]. Disponible à l'adresse : http://ecdc.europa.eu/en/publications/Publications/Dataquality-monitoring-surveillance-system-evaluation-Sept-2014. pdf.

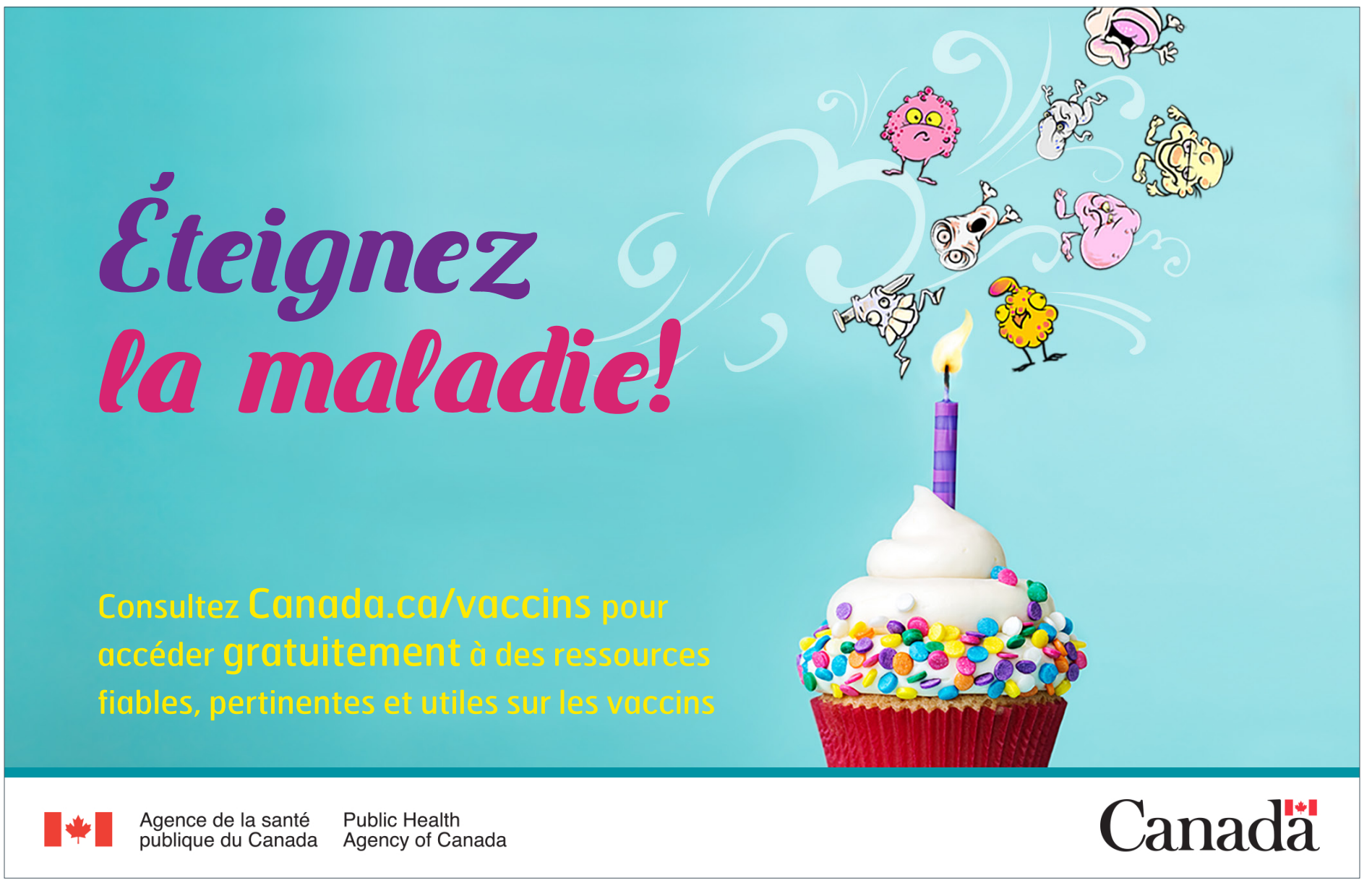

